\title{
Photon Frequency Shift Caused by Gravity and Its Electromagnetic Process
}

\author{
Hans W. Giertz \\ Uppsa Research, Gnesta, Sweden \\ Email: hans@miklagaard.com
}

Received 20 March 2014; revised 20 April 2014; accepted 27 April 2014

Copyright (C) 2014 by author and Scientific Research Publishing Inc.

This work is licensed under the Creative Commons Attribution International License (CC BY). http://creativecommons.org/licenses/by/4.0/

(c) (i) Open Access

\begin{abstract}
In the present paper, photon frequency shift, e.g. gravitational red and blueshift, is described as the electromagnetic influence from gravity waves on photons, e.g. light waves. Previous reports have described the dynamic electromagnetic processes of the atom, the photon and gravity, and how these characteristics are measured. Results from these reports have been compiled into a theoretical model. The theoretical model describes the mechanism which results in photon frequency shift caused by a gravitational field. The theoretical model gives results similar to General Relativity using Schwarzschild metric. The theoretical model is mapped on black holes.
\end{abstract}

\section{Keywords}

Gravitational Redshift, Gravitational Blueshift, Gravity, Photon, Schwartzschild Metric, Black Hole

\section{Introduction}

State of the art science offers no explanation concerning the mechanism which results in gravity and no description of the mechanism which results in light and photons. Gravity and photons are described as more or less undefined particles. It is a cumbersome task to describe astrophysics based on undefined mechanisms and poorly defined particles.

Novel research provides a fundamentally new description of the atom [1], its forces including gravity [2] and its energy including emitted photons [3], based on electromagnetic theory. This has resulted in a theoretical model and increased understanding of gravitational lensing [4]. The present paper uses the same definition of gravity and photons and reuses the structure of the theoretical model [4], and this results in a theoretical model of gravitational red and blueshift. Consequently, a novel definition of gravity and photon provides simple solutions to unsolved astrophysical phenomena.

It has been reported that the universe contains a gigantic singularity which generates very low frequency and 
synchronized TEM (transverse electromagnetic) waves, having extremely high amplitude and energy [1]-[3]. Atoms contain oscillators with different natural frequencies. These oscillators absorb TEM waves at specific frequencies. The absorbed electromagnetic energy accounts for atomic internal and external forces as well as atomic stored energy and emitted energy [1]-[3]. These TEM waves are impossible to measure using state of the art methods. However, a novel method enables characterization of TEM waves emitted by the singularity, absorbed by atoms and re-emitted by atoms [1]-[3].

It has also been reported that atoms absorb these TEM waves in the frequency range $0.005-0.03 \mathrm{~Hz}$ [1]. The absorbed energy is very large and may account for atomic forces and atomic stored energy.

Furthermore, it has been reported that atoms also absorb TEM waves with frequency $69.9 \mathrm{~Hz}$ [2]. These TEM waves are called gravity waves in the present paper. These gravity waves are then re-emitted uniformly into space, whereby some are absorbed by other atoms. This creates a mutual flow of synchronized TEM waves between atoms, resulting in a mutual force of attraction, i.e. gravity.

It has also been reported that excess energy quanta $h v$ in oscillators, contained in a particle, an electron or an atom, is superpositioned on gravity waves absorbed by the oscillator. The re-emitted TEM wave has the superpositioned energy quanta $h v$ and the frequency $69.9 \mathrm{~Hz}+v$ [3]. The superpositioned energy quantum $h v$ is called photon and it explains the photon-wave duality.

The present study shows that gravity waves, i.e. TEM waves with frequency $69.9 \mathrm{~Hz}$, radiated from a gravitational body, interact with TEM waves (with frequency $69.9 \mathrm{~Hz}$ ) contained in light waves. Energy is transferred between the TEM waves, and which influences the light wave's superpositioned energy quanta $h v$. Energy is transferred to the light wave when the light wave propagates towards the gravitational body, resulting in that $h v$ and $v$ increase, i.e. blueshift. Energy is transferred from the light wave when the light wave propagates from the gravitational body, resulting in that $h v$ and $v$ decrease, i.e. redshift [5].

The objective with the present paper is to build on results already presented in reports [1]-[4]. This information is compiled into a theoretical model in Section 2, which describes the mechanism behind gravitational red and blueshift. The theoretical model is based on state of the art electromagnetic theory [6], the Standard Model [7], wave theory [8], superposition principle [9] and photon theory [10]. The present theoretical model is compared with classical interpretation of redshift based on General Relativity and Schwarzschild metric [11]-[13]. The present theoretical model is mapped on black holes [14]. Discussion and conclusions are found in Section 3.

The aim of the study is to present a theoretical model of photon frequency shift caused by gravity, e.g. gravitational red and blueshift.

\section{Theoretical Model}

\subsection{Gravity}

The theoretical model builds on the observation that atomic oscillators 69.9 , with natural frequency $69.9 \mathrm{~Hz}$, absorb TEM waves with frequency $69.9 \mathrm{~Hz}$ [2]. The TEM waves originate from a singularity in the universe and have been thoroughly described [1] [2]. The absorbed TEM waves are then re-emitted uniformly into space.

The behavior and characteristics of plane TEM waves is independent of their frequency. The field vectors $\mathbf{E}$ and $\mathbf{B}$ or $\mathbf{H}\left(\mathbf{B}=\mu_{0} \mathbf{H}\right)$ are linked and perpendicular. The field vector amplitudes relate as $E=c B$, where $c$ is the speed of light. The electric and magnetic field vector amplitudes $E$ and $B$ decrease with the distance from the source as $1 / r$. The Poynting vector $\mathbf{S}$ describes the TEM energy flow density [6].

$$
\mathbf{S}=\mathbf{E x H} \text {. }
$$

The energy stored in the magnetic field is just equal to that in the magnetic field. The direction of energy flow is reversed for a wave travelling in the opposite direction because the phase of $\mathbf{E}$ and $\mathbf{H}$ is reversed.

The theoretical model comprises a huge singularity in space, $\mathbf{0}$ in Figure 1. It emits vast amount of plane TEM waves with frequency $69.9 \mathrm{~Hz}$. Its energy flow density at distance $R$ is described by its Poynting vector $\mathrm{S}_{\mathbf{0}}(R)$.

One source, Source 1, as $\mathbf{1}$ in Figure 1, where $R$ is the distance to the singularity, absorbs TEM waves from the singularity described by $\mathbf{S}_{\mathbf{0}}(R)$. Source 1 re-emits $\mathrm{TEM}_{1}$ waves described by its Poynting vector $\mathbf{S}_{\mathbf{1}}(r)$ at the distance $r$ from Source 1. Source 2, as 2 in Figure 1, where $R$ is the distance to the singularity and $r$ is the distance to Source 1 , absorbs TEM waves from the singularity described by $\mathbf{S}_{\mathbf{0}}(R)$. Source 2 re-emits plane TEM waves described by $\mathbf{S}_{2}(r)$ uniformly into space. In the direction towards Source 1 plane $\mathrm{TEM}_{1}$ and $^{\mathrm{TEM}} \mathrm{M}_{2} \mathrm{waves}$ 


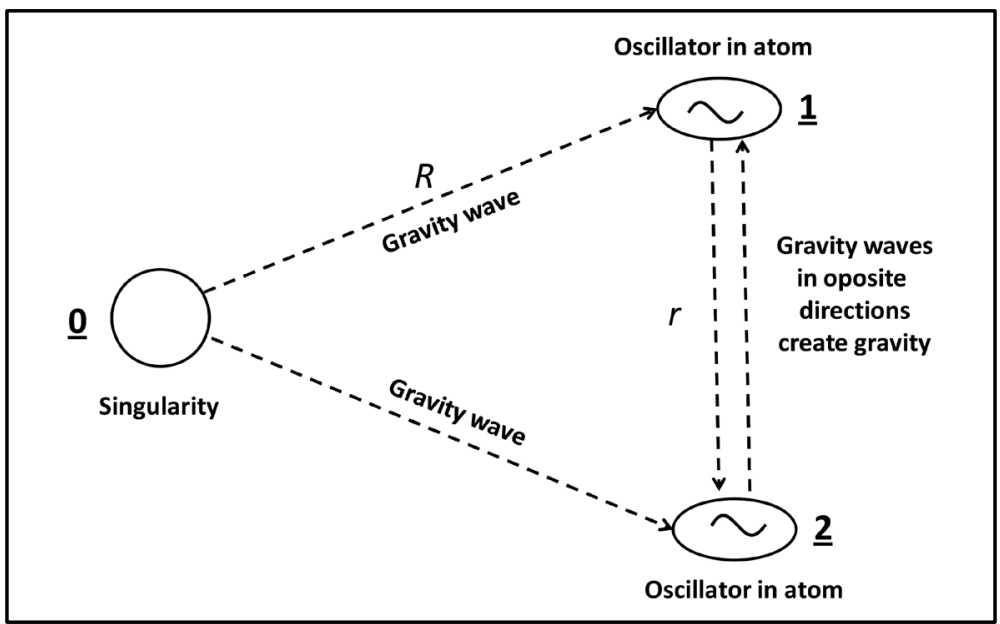

Figure 1. A singularity $\underline{\mathbf{0}}$ generates gravity waves (low frequency plane TEM waves). Oscillators ${ }_{69.9} \underline{\mathbf{1}}$ and $\underline{\mathbf{2}}$ in atoms create resonance with gravity waves and re-emit them unaltered. This causes flow of gravity waves between atoms and their oscillators $69.9 \underline{\mathbf{1}}$ and $\underline{\mathbf{2}}$. The flow of gravity waves in opposite directions between oscillators 69.9 results in mutual force of attraction, i.e. gravity.

interact because of the force between their synchronized field vectors. This also implies that there is energy transfer from $\mathrm{TEM}_{1}$ to $\mathrm{TEM}_{2}$ when $\mathrm{TEM}_{2}$ propagates towards Source 1. In equilibrium there is energy transfer in both directions, i.e. from $\mathrm{TEM}_{1}$ to $\mathrm{TEM}_{2}$ vice versa. Note that $\mathbf{E}$ and $\mathbf{H}$ are always perpendicular and hence $\mathbf{S}=$ ExH can be replaced by $S=E \cdot H$. Equilibrium is defined by the amount of energy that Source 1 is able to deliver at Source 2, e.g. at the distance $r, S_{1}(r)$, and the amount of energy that Source 2 is able to deliver at Source 1, i.e. $S_{2}(r)$. This results in back reaction force $F(r)$, i.e. radiation reaction [11]. This creates mutual force of attraction $F(r)$ :

$$
F(r)=\gamma \cdot S_{1}(r) \cdot S_{2}(r) .
$$

It is here assumed that the distance $R$ to the singularity is constant. Hence, $\mathbf{S}_{\mathbf{0}}(R)$ is constant and thus $\gamma$ is a constant.

The entity which absorbs and re-emits TEM waves with frequency $69.9 \mathrm{~Hz}$ is an (forced damped) oscillator 69.9 with natural frequency $69.9 \mathrm{~Hz}$. It is now assumed that each source consists of many oscillators ${ }_{69.9}$ where each atomic oscillator $_{69.9}$ re-emits TEM waves described by its Poynting vector $\delta S(r)$ and where $r$ denotes the distance from the source and its oscillator ${ }_{69.9}$. $\mathbf{E}$ and $\mathbf{H}$ decrease linearly with the distance $r$ implying that $\delta S(r)$ decreases with the square of the distance; $\delta S(r)=\delta S(0) / r^{2}$. The mutual force of attraction $\delta F(r)$ between two atomic oscillators $_{69.9}$ with distance $r$ is

$$
\delta F(r)=\gamma \cdot \delta S(0) \cdot \delta S(0) / r^{2} .
$$

In order to simplify $\delta S(0)$ is from now on denoted $\delta S$.

It is now assumed that one oscillator $_{69.9}$ acts on $p$ collocated oscillators 69.9 , belonging to the set $\mathbf{P}$ of all oscillators $_{69.9}$ at Source 2, and where each force can be described by $\delta F(r)$. These oscillators 69.9 are synchronized resulting in the force

$$
\sum_{p \in P} \delta F(r)=\gamma \cdot S \cdot \sum_{p \in P} \delta S / r^{2}=\gamma \cdot(\delta S \cdot \delta S) \cdot p / r^{2} .
$$

It is now assumed that $n$ collocated oscillators $_{69.9}$, belonging to the set $\mathbf{N}$ of all oscillators 69.9 at Source 1 , act on $p$ collocated oscillators ${ }_{69.9}$, belonging to the set $\mathbf{P}$ of all oscillators ${ }_{69.9}$ at Source 2, resulting in the total force $F(r)$ :

$$
F(r)=\gamma \cdot \sum_{n \in N} \delta S \cdot \sum_{p \in P} \delta S / r^{2}=\gamma \cdot(\delta S \cdot \delta S) \cdot n \cdot p / r^{2} .
$$

$\delta S \cdot \delta S$ is a constant described by the intrinsic characteristics of the oscillator 69.9 and $S_{0}(R)$, therefore Equ- 
ation (5) is simplified into

$$
F(r)=\xi \cdot n \cdot p / r^{2}
$$

where $\xi$ is a constant.

In a previous study Equation (6) was called the law of gravity between two clusters, at distance $r$ containing $n$ respectively $p$ oscillators $_{69.9}$ [2]. Note that the cluster can be few oscillators ${ }_{69.9}$, an electron, an atom, a mass, a planet or a black hole.

Equation (6) can be illustrated with gravity between the earth and the sun. The earth contains $n$ atomic oscillators $_{69.9}$ creating a force $n \cdot \delta F(r)$ on every atomic oscillator ${ }_{69.9}$ in the sun. The sun contains $p$ oscillators 69.9 , thus the total force of attraction is $n \cdot p \cdot \delta F(r)$ and that is equal to Equation (6). The earth's mass $M_{1}$ is proportional to the number of atomic oscillators 69.9 on earth, i.e. $M_{1} \sim n$, and the sun's mass $M_{2} \sim p$. Equation (6) is then approximately equal to the Newtonian geometric law of gravity, where $F_{G}$ is the gravitational force:

$$
F_{G}=\gamma \cdot S_{1}(0) \cdot S_{2}(0) / r^{2}=\xi \cdot n \cdot p / r^{2} \approx F_{\text {Newton }}=G M_{1} M_{2} / r^{2} .
$$

$S_{1}(0)$ is the Poynting vector of Source 1 or body 1 and $S_{2}(0)$ is the Poynting vector of Source 2 or body 2 . $\xi, G, S_{1}(0)$ and $S_{2}(0)$ in Equation (7) depend on the distance $R$ between the singularity and body 1 respectively body 2 .

\subsection{Photon}

The theory of photon energy transport in space can be illustrated with light waves. It has been shown [3] that light waves are the result of the sum of time limited processes, i.e. acceleration or deceleration of oscillators 69.9 in an electron or atomic nucleus, see Figure 2. These oscillators 69.9 absorb and re-emit gravity waves with frequency $69.9 \mathrm{~Hz}$. Accelerating or decelerating an electron or an atomic nucleus implies that they gain energy, i.e. each of their intrinsic oscillators ${ }_{69.9}$ increases its energy and this excess energy can be described by one or many energy quanta $h v$. In this case the oscillator $_{69.9} \underline{4}$ in the electron or atom contains one energy quantum $h v$ at a time, where $h$ is Planck constant or quantum of action.

The energy quantum $h v$ is discrete and limited in time. The energy quantum $h v$ is superpositioned on a reemitted TEM wave. This TEM wave contains the superpositioned energy quantum $h v$ and the frequency $69.9 \mathrm{~Hz}$ and instant frequency $v$. Hence, the re-emitted wave is a gravity wave with superpositioned electromagnetic energy. The superpositioned electromagnetic energy quantum $h v$ with frequency $v$ is the photon. In a continuous process the sum of many photons and their energy quanta $h v$, superpositioned on re-emitted gravity waves, result in light waves.

The light wave's amplitude is proportional to the number of photons per time unit, which is proportional to the number of involved energy quanta $h v$, which in its turn is proportional to the number of involved oscillators $_{69.9}$. This light wave has the frequency $69.9 \mathrm{~Hz}+v$.

The light wave can be absorbed in matter $\underline{\mathbf{5}}$ and where the superpositioned energy quanta $h v$ are absorbed, resulting in that the light wave is converted back to the original gravity wave with frequency $69.9 \mathrm{~Hz}$.

The energy contained in a TEM wave is proportional to its frequency $v$. From this follows that the superpositioned energy and the photon energy $\delta E$ is:

$$
\delta E=\hbar \omega=h \nu .
$$

The TEM wave's superpositioned energy and momentum are related as $\delta E=p c$, where $p$ is the magnitude of the momentum vector $\mathbf{p}$. This derives from the following relativistic relation, with $m=0$ [8]:

$$
\begin{gathered}
\delta E^{2}=p^{2} c^{2}+m^{2} c^{4}, \\
\mathbf{p}=\hbar \mathbf{k}
\end{gathered}
$$

where $\mathbf{k}$ is the wave vector (where the wave number $k=|k|=2 \pi v / c$ ), and $\hbar=h / 2 \pi$ is the reduced Planck constant. Since $\mathbf{p}$ points in the direction of the TEM wave propagation, the magnitude of the momentum is:

$$
p=\hbar k=h v / c \text {. }
$$

Thus the photon contained in the light wave is not a particle; however, discrete electromagnetic energy quantum $h v$, with frequency $v$, superpositioned on a low frequency TEM wave, i.e. a gravity wave. From this follows 


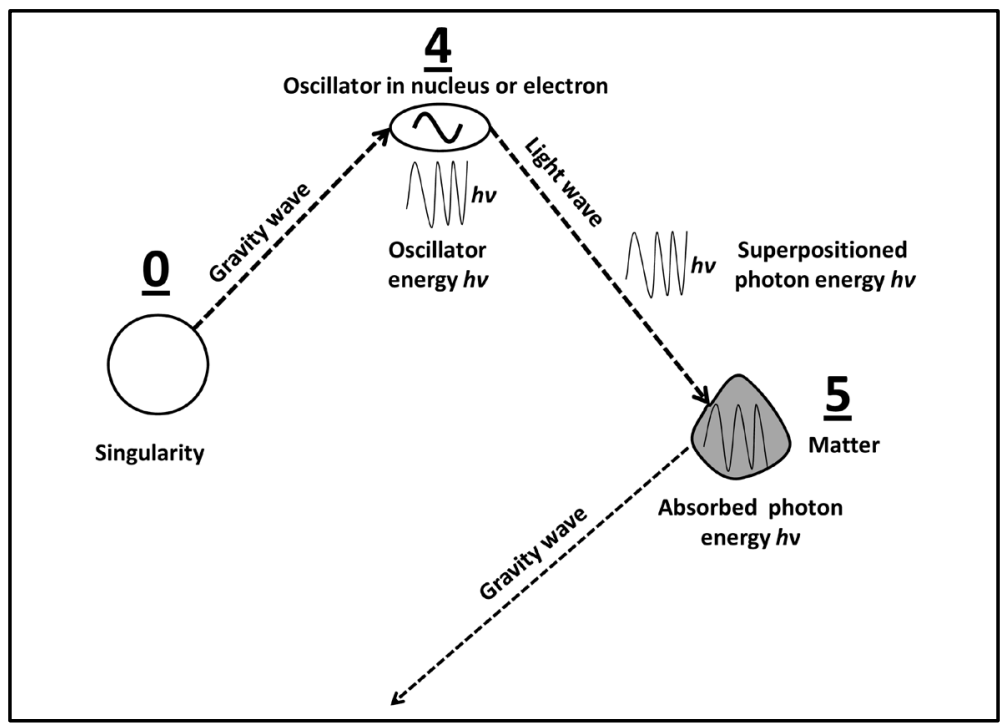

Figure 2. Electrons can be accelerated or decelerated. The electron contains oscillators $_{69.9}$. The oscillator $_{69.9} \underline{\boldsymbol{4}}$ in an electron creates resonance with a gravity wave and excess energy $h v$, caused by electron acceleration deceleration, is superpositioned on the re-emitted wave. The superpositioned energy $h v$ with frequency $v$ represents the photon. This wave with frequency $69.9 \mathrm{~Hz}$ and $v$ is re-emitted as a radio wave. The superpositioned energy $h v$ can be absorbed in matter $\underline{\mathbf{5}}$, whereby the light wave is converted into the original gravity wave with frequency $69.9 \mathrm{~Hz}$.

that the photon is strictly mass less, has zero charge and propagates with the speed of the low frequency TEM wave, i.e. with the speed of light in free space.

\subsection{Classical Gravitational Redshift}

The classical definition of gravitational redshift is based on General Relativity and the Schwarzschild metric. Redshift is often denoted with the dimensionless variable $z$, defined as the fractional change of the wavelength [11]

$$
z=\left(\lambda_{o}-\lambda_{e}\right) / \lambda_{e} .
$$

where $\lambda_{o}$ is the wavelength of the electromagnetic radiation (photon) as measured by the observer. $\lambda_{e}$ is the wavelength of the electromagnetic radiation (photon) when measured at the source of emission. The gravitational redshift of a photon can be calculated in the framework of General Relativity (using the Schwarzschild metric) as

$$
\lim _{r \rightarrow \infty} z(r)=1 / \sqrt{\left(1-r_{s} / r\right)}-1,
$$

with the Schwarzschild radius [12]

$$
r_{s}=2 G M / c^{2} .
$$

where $G$ denotes Newton's gravitational constant, $M$ the mass of the gravitating body, $c$ the speed of light, and $r$ the distance between the center of mass of the gravitating body and the point at which the photon is emitted. The redshift is not defined for photons emitted inside the Schwarzschild radius, the distance from the body where the escape velocity is greater than the speed of light. Therefore this formula only applies when $r$ is at least as large as $r_{s}$. When the photon is emitted at a distance equal to the Schwarzschild radius, the redshift will be infinitely large. When the photon is emitted at an infinitely large distance, there is no redshift. In the Newtonian limit, i.e. when $r$ is sufficiently large compared to the Schwarzschild radius $r_{s}$, the redshift can be approximated by a binomial expansion to become 


$$
\lim _{r \rightarrow \infty} z_{\text {approx }}(r)=1 / 2 \cdot r_{s} / r=G M / c^{2} r .
$$

\subsection{Theoretical Model of Photon Frequency Shift}

According to the present theoretical model the gravitating body emits $\mathrm{TEM}_{1}$ waves where the Poynting vector of the gravitational field, at the distance $r$, is $\mathbf{S}_{\mathbf{1}}(r)$. The light wave (or radio wave, gamma ray) consists of superpositioned energy quanta $h v$ on its $\mathrm{TEM}_{2}$ wave (gravity wave) with frequency $69.9 \mathrm{~Hz}$. The Poynting vector of the $69.9 \mathrm{~Hz}$ light wave component $\left(\mathrm{TEM}_{2}\right)$ at the point of intersection between $\mathrm{TEM}_{1}$ and $\mathrm{TEM}_{2}$ is $\mathbf{S}_{2}$. In the direction towards or from the gravitating body $\mathrm{TEM}_{1}$ and $\mathrm{TEM}_{2}$ waves interact because of the force $\mathbf{F}(r)$ between their synchronized field vectors. $\mathbf{F}(r)$ is described by Equation (2) in vector form

$$
\mathbf{F}(r)=\gamma \cdot \mathbf{S}_{1}(r) \cdot \mathbf{S}_{2},
$$

where $\mathbf{F}(r)$ is positive when $\mathbf{S}_{1}(r)$ and $\mathbf{S}_{2}$ propagate in opposite directions and negative when they propagate in the same direction. This results in energy transfer from $\mathrm{TEM}_{1}$ to the light wave when the light wave propagates towards the gravitating body and energy transfer from the light wave to $\mathrm{TEM}_{1}$ when the light wave propagates from the gravitating body. The transferred energy $\delta E_{\text {transfered }}$ at distance $r$ over a small distance $\delta r$ is implicitly described by Equation (16)

$$
\delta E_{\text {transfered }}(r)=\varsigma \cdot S_{1}(r) \cdot S_{2},
$$

where $\varsigma$ is a constant. $\delta E_{\text {transfered }}(r)$ is positive when $S_{1}(r)$ and $S_{2}$ propagate in opposite directions and negative when they propagate in the same direction.

According to the present theoretical model the system is linear and hence, it applies to the superposition principle [10]. When the light wave propagates towards the gravitating body the positive energy $\delta E_{\text {transfered }}$ is superpositioned on the light wave. Therefore the superpositioned energy results in that the energy quantum $h v$ increases, resulting in that the photon frequency $v$ increases, which is called blueshift in the case of light.

When the light propagates from the gravitating body the negative energy $\delta E_{\text {transfered }}$ is removed from the light wave's superpositioned energy. Therefore the energy in the superpositioned energy quantum $h v$ decreases, resulting in that the photon frequency $v$ decreases, which is called redshift in the case of light. A light wave which is created at the distance $r$ and measured at $r \rightarrow \infty$ experiences redshift described by integrating Equation (17).

Consequently, according to Equation (17) the transferred energy $\delta E_{\text {transfered }}$ and the change in photon frequency $v$ are proportional to $S_{1}(r)$, which decreases as $1 / r^{2}$. However, the total redshift measured at $r \rightarrow \infty$ is the integral of Equation (17) which decreases as $1 / r$. Equation (7) displays that $S_{1}(0)$ is proportional to GM. Consequently, the present theoretical model gives approximately the same redshift as the Schwarzschild metric and Equation (15).

\subsection{Mapping on Black Holes}

A black hole is characterized by its huge mass and extremely large gravitational field [14]. Every photon generated within or close to the black hole experiences extremely large loss of energy according to Equation (17) and will lose all of its energy, i.e. $h v=0$. This is valid for all types of photons, ranging from radio waves to gamma rays.

Light waves, and photons in general, passing the black hole at a sufficiently small distance, will be bent towards and into the black hole as reported previously [4].

The consequence is that no photons escape the black hole, i.e. the emitted energy, which can be measured with state of the art methods, is zero. However, the black hole emits vast amount of gravity, i.e. vast amount of energy which cannot be measured with state of the art methods. Hence, it is only black in the eyes of prevailing science. In reality it is a gravity star.

\section{Discussion and Conclusions}

The strength of the present theory is a novel method facilitating measurement of relevant parameters [1]-[3]. TEM waves originating from the singularity have been measured to a degree which allows description of their characteristics such as direction of origin, amplitude, phase, frequencies and field vector behavior [1]-[3]. Furthermore, TEM waves, absorbed and re-emitted by atoms, have been measured to a degree which reveals the atomic intrinsic mechanism: forced damped oscillators with natural frequencies $0.005-0.03 \mathrm{~Hz}[1]$ and $69.9 \mathrm{~Hz}$ 
[2]. Moreover, photons, ranging from extremely low frequency to high frequency, have been produced and measured in laboratory [3]. The force of attraction between TEM waves has been measured. Hence, crucial parts of the present theory have been measured in laboratory and some parts have been simulated and reproduced in laboratory.

The platform of modern physics is elementary particle physics described in the Standard Model [7]. The Standard Model lacks definition of gravity; hence, it falls short in describing gravitational red and blueshift.

The General Relativity provides a metric description of gravity [11]-[13]. However, it does not define the mechanism creating gravity and the mechanism creating photons.

A new theory has been launched which describes the mechanism creating gravity [2], the mechanism creating photons and light waves [3] and the mechanism which results in energy transfer between gravity waves and superpositioned energy quanta $h v$, i.e. photons, in e.g. light waves. It explains gravitational red and blueshift. The theory is based on electromagnetic theory and wave theory.

\section{References}

[1] Giertz, H.W. (2013) Atoms Absorb Low Frequency Electromagnetic Energy. Open Journal of Microphysics, 3, 115120.

[2] Giertz, H.W. (2013) Gravity Caused by TEM Waves Operating on Dipoles in Atoms. International Journal of Astronomy and Astrophysics, 3, 39-50. http://dx.doi.org/10.4236/ijaa.2013.32A007

[3] Giertz, H.W. (2013) Photons Are EM Energy Superpositioned on TEM Waves. Open Journal of Microphysics, 3, 71-80. http://dx.doi.org/10.4236/ojm.2013.33013

[4] Giertz, H.W. (2014) Gravitational Lensing Described by Its Electromagnetic Processes. International Journal of Astronomy and Astrophysics, 4, 294-300. http://dx.doi.org/10.4236/ijaa.2014.41024

[5] Pound, R.V. and Snider, J.L. (1965) Effect of Gravity on Gamma Radiation. Physical Review, 140, 788-803. http://dx.doi.org/10.1103/PhysRev.140.B788

[6] Melrose, D.B. and McPhedran, R.C. (1991) Electromagnetic Processes in Dispersive Media. Cambridge University Press, Cambridge. http://dx.doi.org/10.1017/CBO9780511600036

[7] Burgess, C. and Moore, G. (2007) The Standard Model: A Primer. Cambridge University Press, Cambridge.

[8] Kneubühl, F.K. (1997) Oscillations and Waves. Springer, Berlin. http://dx.doi.org/10.1007/978-3-662-03468-2

[9] Ostrovsky, L.A. and Potapov, A.I. (2002) Modulated Waves: Theory and Application. Johns Hopkins University Press, Baltimore.

[10] Bialynicki-Birula, I. (1994) On the Wave Function of the Photon. Acta Physica Polonica A, 86, 97-116.

[11] Misner, C.W., Thorne, J.K.S. and Wheeler, A. (1973) Gravitation. W. H. Wheeler, San Fransisco.

[12] Schwarzschild, K. (1916) Über das Gravitationsfeld eines Massenpunktes nach der Einsteinschen Theorie. Sitzungsberichte der Deutschen Akademie der Wissenschaften zu Berlin, Klasse für Mathematik, Physik, und Technik, 189.

[13] Heinzle, J.M. and Steinbauer, R. (2002) Remarks on the Distributional Schwarzschild Geometry. Journal of Mathematical Physics, 43, 1493-1508. http://dx.doi.org/10.1063/1.1448684

[14] Bozza, V. (2010) Gravitational Lensing by Black Holes. General Relativity and Gravitation, 42, 2269-2300. http://dx.doi.org/10.1007/s10714-010-0988-2 